\title{
Collecting duct of Bellini renal carcinoma with psoas muscle recurrence: A case report and review of literature
}

\author{
Michael Erlano Chua, MD; ${ }^{*}$ Karen Olondriz, MD; ${ }^{\dagger}$ Michael Maniwa, MD; Jonathan Mendoza, MD; ${ }^{*}$ \\ Josefino Castillo, $M D^{*}$
}

*St. Luke's Medical Center-Institute of Urology, Philippines; †Department of Preventive and Community Medicine, St. Luke's College of Medicine, Philippines

Cite as: Can Urol Assoc J 2014;8(3-4):e167-71. http://dx.doi.org/10.5489/cuaj.1603 Published online March 11, 2014.

\section{Abstract}

Collecting duct carcinoma (CDC) is a rare type of renal carcinoma with an aggressive course and poor treatment response. Up to date, there are only few reports of CDC, which may be due to unrecognized or unfamiliarity of its features and characteristics. We report a case of CDC in a 63-year-old male who initially presented with localized disease, and developed psoas muscle metastasis 15 months after partial nephrectomy with good surgical margins. No guidelines currently exist for the management of CDC; however, aggressiveness of the disease warrants close surveillance and consideration of adjuvant therapy even in patients with localized tumours. We postulate that CDC in its localized stage can be managed by partial nephrectomy, while preserving renal function; however, it is also important to consider a good oncologic outcome by administering adjuvant therapy, such as chemotherapy, and targeted therapy for a better prognostic outcome.

\section{Introduction}

Bellini duct or collecting duct carcinoma $(C D C)$ is a rare aggressive variant of renal cell carcinoma associated with poor prognosis, which warrants early recognition and management. ${ }^{1}$ However, up to date, only a few investigational studies on the CDC treatment are available, with no established standard management approach. We present a case of CDC, its clinical presentation, pathologic and immunohistochemical features, the course of condition, and its response to treatment with partial nephrectomy and adjuvant therapies.

\section{Case report}

A 63-year-old male, with no known comorbidities, was referred to our institution due to a right renal mass seen on ultrasonography. He initially presented with 2 episodes of painless gross hematuria not associated with dysuria, flank pain, fever or history of urinary tract infection. Physical examination was unremarkable.

A contrast-enhanced abdominal computed tomography (CT) scan confirmed the presence of an enhancing exophytic soft tissue density in the middle pole of the right kidney, measuring $3.5 \times 4.1 \mathrm{~cm}$ (Fig. 1). In-vitro glomerular filtration rate revealed split renal function of $35 \mathrm{~mL} / \mathrm{min}$ and $32.7 \mathrm{~mL} / \mathrm{min}$ on the left and right, respectively. A right partial nephrectomy was performed to maximize renal function preservation without compromising oncologic outcome. Histopathology revealed CDC with capsular and lymphovascular invasion; surgical line of resection and perirenal fat were negative for tumour. Immunohistochemical stains were positive for CK19, CD10 and vimentin (Fig. 2) and negative for HMWCK, CK7 and PAS; these results supported the diagnosis of CDC stage I (T1NOM0). A CT scan done 1 month postoperatively was unremarkable. The patient showed no evidence of disease and was lost to follow-up.

Fifteen months later, the patient returned for a consult due to a dull, persistent and progressive right flank pain radiating to the right lower abdomen. A non-contrast abdominal CT scan revealed a $6.2 \times 6.2 \times 8$-cm the low density collection within the enlarged right psoas muscle (Fig. 3 ). The patient underwent exploratory laparotomy with en bloc excision of the superficial psoas muscle with wide margins. Intraoperatively, we did not notice gross local tumour recurrence on the right kidney or enlarged lymph nodes. Histopathology was consistent with CDC (Fig. 4). Metastatic workup revealed no regional or distant metastasis. Routine quarterly surveillance CT of the abdomen was done for 2 years and showed no recurrence or distant metastasis. The patient was again lost to follow-up.

Two years later, the patient again presented with recurrence of right flank pain with difficulty in right hip flexion. Abdominal CT scan with intravenous contrast revealed an $8.1 \times 10.2 \times 14.4-\mathrm{cm}$ enhancing mass in the right retro- 


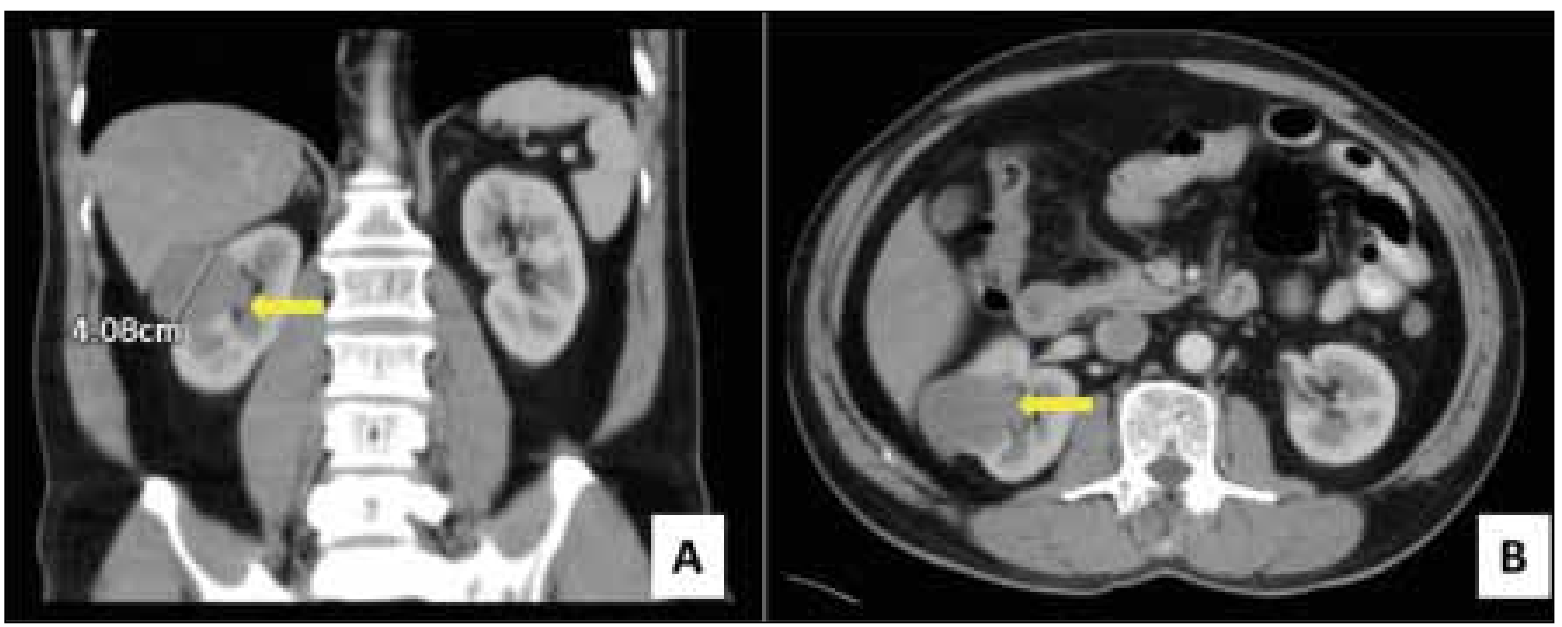

Fig. 1. Abdomino-pelvic computed tomography scan with intravenous contrast (coronal $A$ and axial $B$ views). Yellow arrow points on the right renal mass.

peritoneal space extending into the right psoas and iliacus muscles, with an enlarged external iliac lymph node (Fig. 5, part A). Chest x-ray and bone scan were negative for distant metastasis. Since the patient was hesitant to undergo another surgery, he was given 28 sessions of external beam radiotherapy. On post-radiation therapy, his abdominal CT scan showed an increase in the size of the mass. His chest CT scan demonstrated diffuse pulmonary nodules (Fig. 5, part B). Bone scintigraphy revealed multiple bone metastasis.

The patient was then advised on systemic targeted therapy. Consequently, he was given successive cycles of the sunitinib $50 \mathrm{mg} /$ day, for 4 weeks with 2 weeks off therapy. On the third cycle of chemotherapy, his follow-up CT scan showed an interval decrease in the size of the mass and his bone scintigraphy showed interval resolution of blastic lesions. However, on the ninth cycle of sunitinib, treatment resistance was recognized with progression of the retroperitoneal mass and infiltration into the posterior muscle, skeletal structures and subcutaneous space. The patient started to manifest symptoms of paraneoplastic syndrome. Palliative and supportive management were given. The patient died 5 years and 4 months after the initial diagnosis.

\section{Discussion}

CDC is a rare aggressive type of renal epithelial malignancy, comprising about $1 \%$ to $3 \%$ of all renal neoplasm. ${ }^{2}$ Presently only 270 cases have been reported worldwide. ${ }^{2}$ It is more common in males and in the younger population (58-61 years). Like our patient, most cases present with gross hematuria, a renal mass or flank pain. ${ }^{1}$ Findings on CT include medullary location, heterogenous enhancement and infiltra-

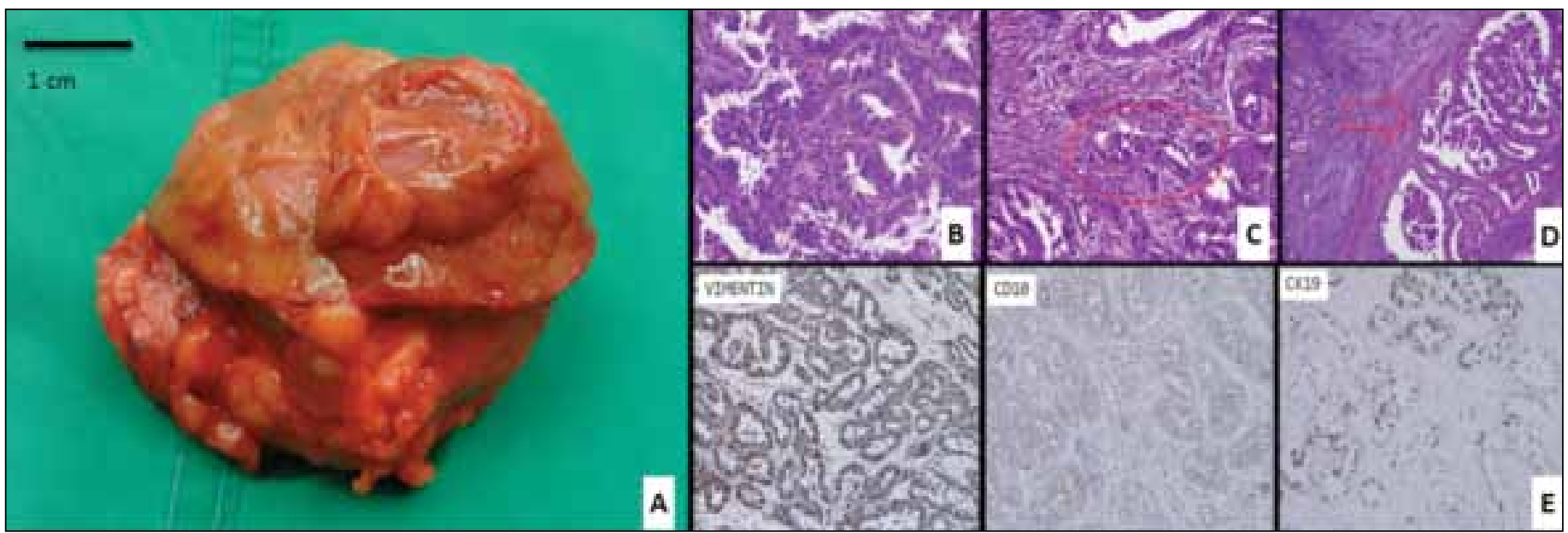

Fig. 2. Box A: Renal mass view from parenchymal line of resection. Blue arrow points to the internal aspect of the renal mass with cream tan and rubbery texture. Yellow arrow point to the cream to pink tan smooth capsule with perirenal fat. Boxes B to D: Hematoxylin and eosin stain on high power view (microscopic magnification $=40 \times$ objectives) showing (Box B) prominent infiltrating tubulopapillary pattern with high grade nuclear atypia and extensive stromal dysplasia. Box C: Red highlight showing lymphovascular invasion. Box D: Red arrow pointing at renal capsule with tumour invasion. Box E: Immunohistochemistry stain: positive for vimentin, CD10 and CK19. 


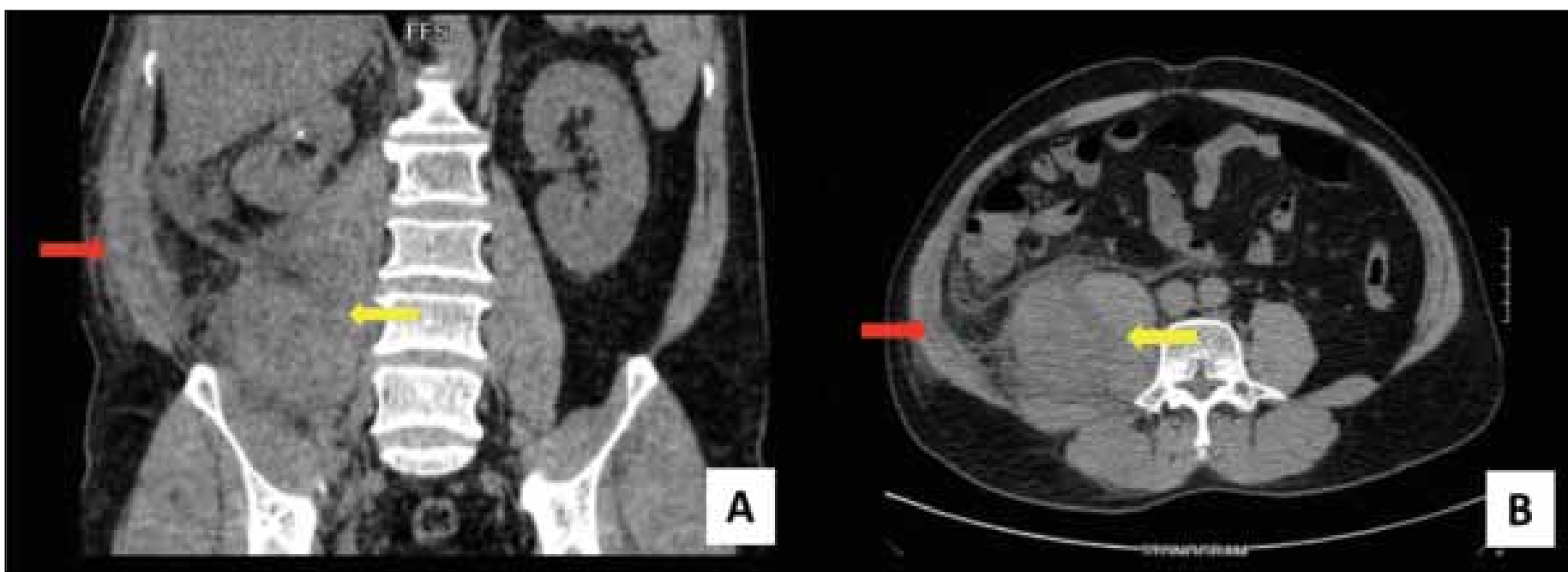

Fig. 3. Abdomino-pelvic computed tomography scan (coronal A and axial B views): Red arrow points on subcutaneous fat stranding. Yellow arrow showing the low density collection within the enlarged right psoas muscle.

tive renal sinus involvement with preserved renal contours and some cystic component. ${ }^{1}$ Rarely, an exophytic characteristic is noted, as reported in this case. Most cases are detected at pT2-pT3 with perinephric extension. ${ }^{1,2}$

In most countries, CDC appears to be unreported since its differentiation requires extensive histopathologic and immunohistochemical examinations. ${ }^{2} \mathrm{CDC}$ is a high-grade renal adenocarcinoma arising in the medulla of the kidney with a prominent infiltrating tubulopapillary pattern exhibiting high-grade nuclear atypia and extensive stromal dysplasia. ${ }^{1,2}$ Immunohistochemical markers include lectin Ulex europaeus, epithelial membrane antigen, and high-molecular weight cytokeratins. Vimentin reactivity is variable. In our case, histologic characteristics with such stains were used to diagnose CDC. ${ }^{3}$
Of all renal neoplasms, CDC has the worst prognosis with most patients eventually developing metastatic disease..$^{1,2}$ Our case initially presented as localized disease, which was completely excised by partial nephrectomy; however, it had metastasized. In our case, the metastasis was first noted in the psoas muscle without prior invasion to the regional lymph nodes. In the case series of CDC by Tokuda and colleagyes, only 1 patient developed psoas metastasis. ${ }^{1}$ The mechanism can occur either by direct invasion or by hematogenous spread, with the latter assumed to be the proposed means of metastasis in our patient since subsequent follow-ups showed no recurrence on the residual parenchyma and renal bed. ${ }^{4}$

Among clinically localized renal cell carcinoma, there is controversy on the effect of microvascular tumour invasion,

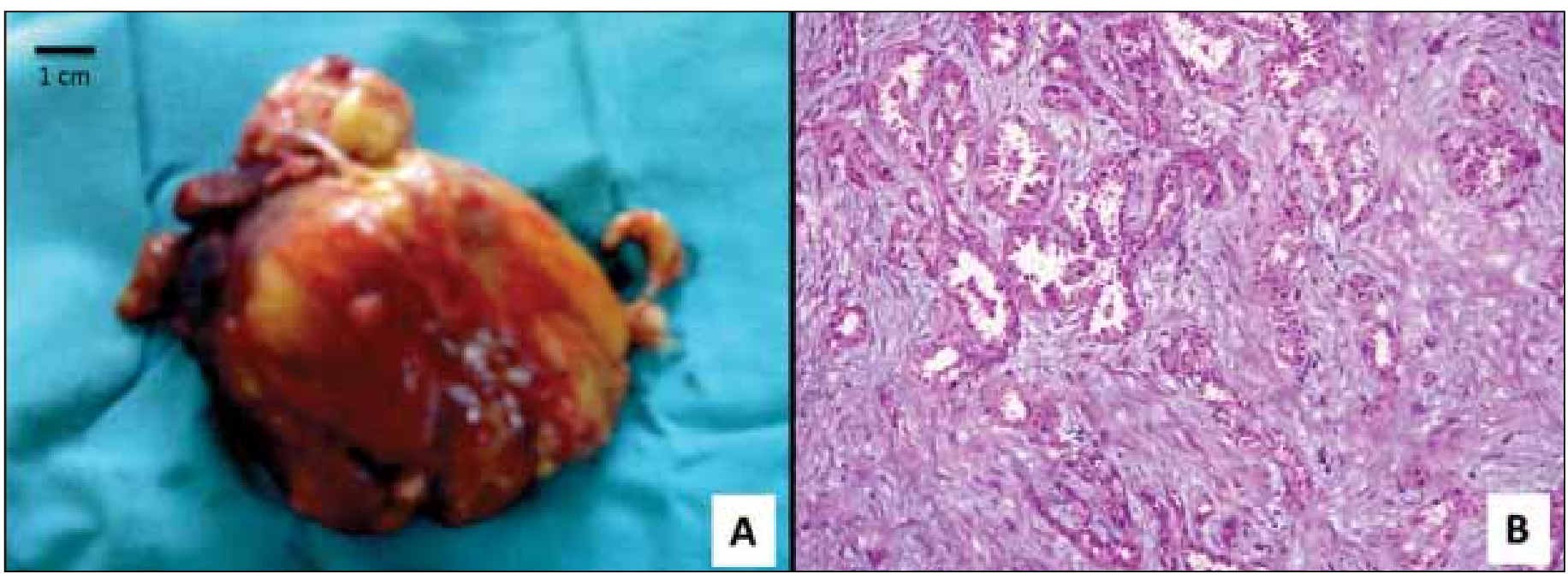

Fig. 4. A: Gross appearance of the mass overlying psoas muscle with yellow arrow pointing to superficial psoas muscle tissue. B: Hematoxylin and eosin stain on high power view (microscopic magnification $=40 \times$ objectives). Yellow arrow points on tubular pattern with atypia and dysplasia consistent with collecting duct carcinoma. 
Chua et al.

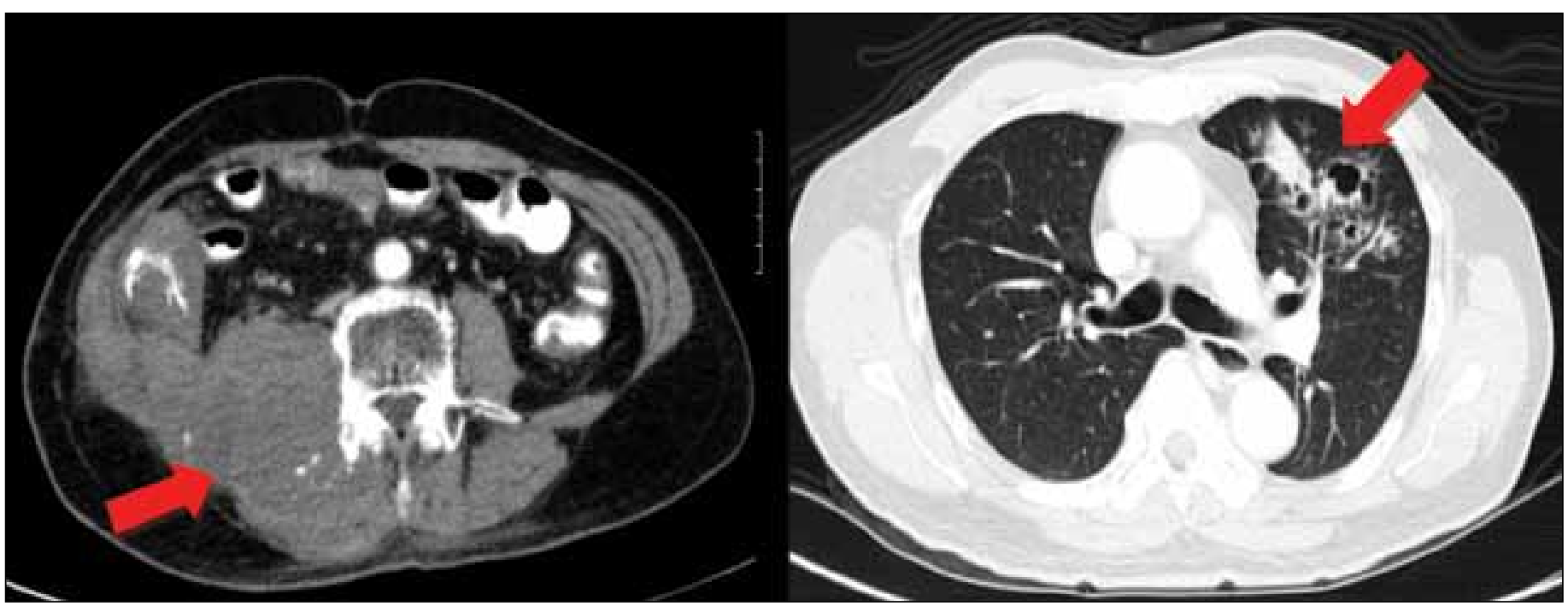

Fig 5. A: Abdominopelvic computed tomography scan with intravenous contrast (axial view). Heterogenously enhancing mass extending from right pararenal space into right psoas and iliacus muscles. B: Chest computed tomography scan with intravenous contrast (axial view). Red arrow points to the heterogenously enhancing diffused calcified and non-calcified pulmonary nodules.

ranging from its clinically insignificant influence to it being the most relevant prognosticator of patient survival. ${ }^{5}$ More studies however have shown that negative vascular invasion is an independent predictor of disease-free survival among patients with clinically localized tumours and post-radical nephrectomy. ${ }^{5}$ To the best of our knowledge, this is the first reported T1N0MO CDC case with clear resection margins that subsequently developed psoas metastasis. We postulate that the presence of lymphovascular invasion could have been responsible for recurrence despite the surgery. Therefore, there is a need for further investigation of the relevance of lymphovascular invasion in CDC prognostication.

There are no existing guidelines for the treatment of CDC and only a few retrospective studies have investigated the significance of potential treatments., ${ }^{1,2}$ The benefit of partial nephrectomy is achieving good oncologic outcome, while preserving renal function such as in this case. Reports show good response with partial nephrectomy, with patients surviving 24 months without evidence of recurrence or metastasis. ${ }^{6}$ Our patient developed metastasis in 15 months, a rare and unanticipated form of disease progression for a CDC stage I tumour. Our case indicates that the aggressive course of CDC should also be expected in early-stage tumours, even after a well-performed nephrectomy. Meticulous surveillance should be made 2 years after the postoperative period.

Due to its limited therapeutic options in CDC and urothelial carcinoma, radiotherapy has been used for control of local recurrence. ${ }^{7}$ However, as described in our patient, radiotherapy does not prevent tumour progression. Sunitinib, a multiple tyrosinase kinase inhibitor, has shown to have observable oncologic benefit in non-clear cell renal carcinoma, specifically CDC. ${ }^{8}$ The 9 cycles of sunitinib given to our patient provided a good initial response; however, it later showed treatment resistance. This is congruent with other studies. ${ }^{9}$ It seems natural that non-clear cell renal carcinoma takes on an alternate biochemical mechanism for tumour proliferation. Recent studies have suggested that the combination of gemcitabine and cisplatin provides an objectively good response for patients with CDC. ${ }^{1,2}$ Therefore, our proposition is to further investigate the role of sunitinib combined with gemcitabine and cisplatin to prevent development of resistance in the treatment of CDC.

\section{Conclusion}

This case highlights the clinicopatholical characteristics, aggressiveness and poor prognosis of CDC. We postulate that $\mathrm{CDC}$, in its localized stage, can be managed by partial nephrectomy while preserving renal function. It is also important to consider a good oncologic outcome by administering adjuvant therapy, such as chemotherapy, and targeted therapy for a better prognostic outcome.

Acknowledgements: Dr. Gylmer Jude Maranon, who served as our external peer review on our final draft and gave us comments and suggestions in improving our manuscript.

Competing interests: Dr. Chua, Dr. Olondriz, Dr. Maniwa, Dr. Mendoza and Dr. Castillo all declare no competing financial or personal interests.

This paper has been peer-reviewed. 


\section{References}

1. Tokuda N, Naito S, Matsuzaki O, et al. Japanese Society of Renal Cancer. Collecting duct (Bellini duct) renal cell carcinoma: a nationwide survey in Japan. J Urol 2006;176:40-3. http://dx.doi.org/10.1016/ S0022-5347(06)00502-7

2. Dason $S$, Allard C, Sheridan-Jonah A, et al. Management of renal collecting duct carcinoma: A systematic review and the McMaster experience. Curr Oncol 2013;20:e223-32. http://dx.doi.org/10.3747/ co.20.1230

3. Vecchione A, Galetti TP, Gardiman M, et al. Collecting duct carcinoma of the kidney: An immunohistochemical study of 11 cases. BMC Urol 2004;4:11. http://dx.doi.org/10.1186/1471-2490-4-11

4. Taira $\mathrm{H}$, Ishii $\mathrm{T}$, Inoue $\mathrm{Y}$, et al. Solitary psoas muscle metastasis after radical nephrectomy for renal cell carcinoma. Int J Urol 2005;12:96-7. http://dx.doi.org/10.1111/j.1442-2042.2004.00976.x

5. Dall'Oglio MF, Antunes AA, Sarkis AS, et al. Microvascular tumour invasion in renal cell carcinoma: The most important prognostic factor. BJU Int 2007;100:552-5. http://dx.doi.org/10.1111/i.1464410X.2007.07015.x
6. Matsumoto H, Wada T, Aoki A, et al. Collecting duct carcinoma with long survival treated by partial nephrectomy. Int J Urol 2001;8:401-3. http://dx.doi.org/10.1046/j.1442-2042.2001.00321.x

7. Maestroni $U$, Ferretti $S$, Dinale $F$, et al. A renal cancer with intermediate characteristics between collecting (Bellini) duct carcinoma and urothelial carcinoma: Case report and review of the literature. Tumori 2006; $92: 545-8$.

8. Procopio $G$, Verzoni E, lacovelli $R$, et al. Is there a role for targeted therapies in the collecting ducts of Bellini carcinoma? Efficacy data from a retrospective analysis of 7 cases. Clin Exp Nephrol 2012; 16:464-7. http://dx.doi.org/10.1007/s10157-012-0589-3

9. Singer EA, Bratslavsky $G$, Linehan WM, et al. Targeted therapies for non-clear renal cell carcinoma. Target Oncol 2010;5:119-29. http://dx.doi.org/10.1007/s1 1523-010-0148-3

Correspondence: Dr. Michael Erlano Chua, St. Luke's Medical Center-Institute of Urology, Philippines; auhc_ekim@yahoo.com 